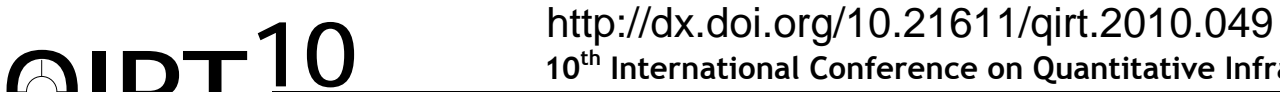 \\ $10^{\text {th }}$ International Conference on Quantitative InfraRed Thermography \\ July 27-30, 2010, Québec (Canada)
}

\section{New approach on determination of emissivity fields using bicolor reflectometry technique}

\author{
by R.Gilblas ${ }^{1,2}$, T. Sentenac ${ }^{1,2}$, D. Hernandez ${ }^{3}$ and Y. Le Maoult ${ }^{1,2}$ \\ ${ }^{1}$ Université de Toulouse; INSA, UPS, Mines Albi, ISAE ; ICA (Institut Clément Ader); F-81013 Albi cedex 09, France \\ ${ }^{2}$ Ecole Mines Albi ; Campus Jarlard, F-81013 Albi, France. \{rgilblas, sentenac,lemaoult\}@mines-albi.fr, \\ ${ }^{3}$ Procédés, Matériaux et Energie Solaire (PROMES), Centre National de la Recherche Nationale (CNRS), B.P. 5, \\ 66125 Font-Romeu Cedex, France. Daniel.Hernandez@promes.fr
}

\begin{abstract}
In a context of quantitative thermography, the main problem for measuring a true temperature field is the knowledge of the thermo-optical properties of the object. The article introduces an approach for measuring the true temperature based on the fusion of the radiance temperature and the measurements in-situ of the material properties. The method is called thermoreflectometry and it is inspired of the work on punctual true temperature measurement by reflectometry presented in [8] on pyroreflectometry.
\end{abstract}

\section{Introduction}

In many industrial processes, an accurate on-line temperature measurement on objects under process, is the key parameter for the optimization and the quality assurance. For temperature measurements, many sensors are available, from the classical thermocouple for acquiring sparse measurements, up to non contact sensors like pyrometers or infrared cameras, required to measure a dense temperature field. As in many cases, contact temperature measurements are difficult, many applications involve non-contact temperature measurement techniques. However, they only provide a radiance temperature. The computation of true temperature of the observed object require the knowledge of optical properties of the surface, like emissivity. Unfortunately, its value is difficult to obtain from the data of the literature or from preliminary measurements because this property depends on several parameters : temperature, direction of the radiation, wavelength, shape and physical properties of the surface.

In the thermography topic, some dedicated techniques were then developed to decrease the effect of the emissivity by measurement in the ultraviolet band [1] or to compensate for its influence by two-colour or multi-colour analysis of the radiation [2] for grey body (constant emissivity in a narrow bandwidth). For every other case, assumptions about the emissivity variations as a function of the wavelength and the temperature have to be ascertained and an emissivity estimation 'in situ' conditions is required. The domain of pyrometry only provides emissivity by indirect determination techniques like measurement of the reflectivity [3] or the absorption [4], the analysis of the radiation emitted and reflected using polarization [5], and the use of reflecting module to increase the apparent emissivity [6]. Unfortunately, the proposed systems are single point temperature evaluations and are often limited by several constraints: optical axis orthogonal to the surface, sensor-surface distance not larger than some centimetres. However, a technique called two-colour pyroreflectometry $[7,8]$ is a potential technique to measure 'in situ' true temperature considering the reflectivity parameter. For opaque materials, an indirect emissivity measurement can be provided by the pyroreflectometry technique and adapted for determining both the radiance temperature and the emissivity from camera measurements.

This article presents an original technique for measuring the true temperature field, called thermoreflectometry, by merging on line the radiance temperature and the emissivity measurements. The technique uses NIR-sensitive InGaAs camera for measuring radiance temperature. The determination of emissivity fields raises again on a reflectometry method which is an active technique based on lasers. The reflectivity data are also acquired in the NIR spectral band. For measuring both radiance temperature and reflectivity fields, the NIR spectral band is the best trade-off due to the high temperature range and the lower dependence to emissivity.

The present paper is organized as follows : the first part presents the theoretical basis of the reflectometry technique. Through the second part, the characteristics of the apparatus are exposed and finally the results, experimental errors, accuracy and its limits are discussed.

\section{Nomenclature}

$L \quad$ Photonic radiative intensity, photons. $s r^{-1} \cdot s^{-1} \cdot m^{-3}$

$L^{0} \quad$ Black body's radiative intensity, photons. $s r^{-1} \cdot \mathrm{s}^{-1} \cdot \mathrm{m}^{-3}$

$u, v$ Pixel coordinates

$\varepsilon^{i} \quad$ Spectral directional emissivity

$\begin{array}{ll}\lambda & \text { Wavelength, } m \\ \lambda_{x} & \text { Effective wavelength, } m\end{array}$


$T \quad$ True Temperature, $K$

$T_{L} \quad$ Radiance temperature, $K$

$T_{C} \quad$ Colour Temperature, $K$

$T^{\star} \quad$ Convergence temperature, $K$

$\mathrm{C}_{1} \quad$ First Planck constant $=1,19.10^{8} \mathrm{~m}^{3} \mathrm{~s}^{-1}$

$\mathrm{C}_{2}$ Second Planck constant $=1,44 \cdot 10^{4} \mathrm{~m} . \mathrm{K}$

$\rho^{\mathrm{i}, \mathrm{n}} \quad$ Directional hemispheric reflexion coefficient $\rho^{\mathrm{i}, \mathrm{r}} \quad$ Bidirectional reflectivity $\left(\mathrm{sr}^{-1}\right)$

$f^{i, r, r 0} \quad$ Normed diffusion indicator

$\eta_{d}{ }^{i, r o} \quad$ Diffusion factor

$I_{D} \quad$ Camera video signal, $N N$

i,r Incidence and reflexion directions

$r_{0} \quad$ Observation direction

$\mathrm{K}_{\text {ref }} \quad$ Reflectivity calibration coefficient, $N N^{-1} S r^{1}$

\section{Theoretical basis of thermoreflectometry technique}

2.1.

Main problem with radiative temperature measurements

The main difficulty of temperature measurement addressed in a radiative context is the measurement of true temperature. Non contact temperature measurements are based on the analysis of thermal radiations emitted by an object. For a black body source, the photonic Planck's law (Eq. (1)) describes this phenomenon.

$$
L^{O}\left(\lambda, T_{L}, u, v\right)=\frac{C_{1} \lambda^{-4}}{\exp \left(\frac{C_{2}}{\lambda T_{L}}\right)-1}
$$

The equation shows that the black body temperature, called radiance temperature, is immediately determined from a radiometer viewing the surface emitted radiative intensity.

For a real object, the radiometer always measures the luminance emitted by the surface object which is equal to the product of the emissivity by the radiative intensity from a black body at the same temperature and the same wavelength. The product is also equal to the black body radiative intensity at a radiance temperature. Eq. (2) sums up the relation:

$$
L^{i}(\lambda, T, u, v)=L^{0}\left(\lambda, T_{L}^{i}, u, v\right)=\varepsilon^{i}(\lambda, T, u, v) L^{\cdot}(\lambda, T, u, v)
$$

The radiometer always indicates a radiance temperature refered to the spectral black body radiative intensity. The true temperature can be only obtained by an additional measurement of emissivity. Our approach is based on an indirect active method using measurement of the surface reflectivity.

\subsection{Emissivity measurement using reflectometry technique}

For opaque materials, following Kirchhoff's laws, the directional emissivity is directly connected to the directional hemispheric reflectivity (see $\mathrm{Eq} 3$ ). The directional hemispheric reflectivity is the integral of all the bidirectional reflectivities on the upper hemisphere (see Eq. 4).

$$
\varepsilon^{i}(\lambda, T, u, v)=ı-\rho^{i, \cap}(\lambda, T, u, v)
$$

$$
\rho^{i, \cap}(\lambda, T, u, v)=\int_{\Pi_{r}=r \pi} \rho^{i, r}(T, \lambda, u, v) \cos \theta_{r} d \Omega_{r}
$$

The bidirectional reflectivity is the ratio between the surface reflected radiative intensity on direction $r$ and the incident intensity on an incident direction $i$. The directional hemispheric reflectivity measurement involves the measurement of bidirectional reflectivities in all directions. Its measurement is usually used in laboratory [9] but its adjustment is difficult on board radiometers.

In general terms, the idea of the method is a reflectivity measurement for a unique angle and the introduction of a relation between the spatial reflectivity behaviour for different angles. The relation is expressed in Eq. (5) and includes the diffusion indicator which represents the values of the bidirectional reflectivities normalized by the reflectivity value for the direction of observation $r_{0}$.

$$
f^{i, r, r .}(\lambda, T, u, v)=\frac{\rho^{i, r}(\lambda, T, u, v)}{\rho^{i, r_{0}}(T, \lambda, u, v)}
$$


Hence, a diffusion factor is defined (see Eq. (6)) as the integral of the diffusion indicator on the upper hemisphere, which represents the volume of the normalized directional hemispheric reflectivity.

$$
\eta_{d}^{i, r_{0}}(\lambda, T, u, v)=\frac{\int_{\Pi_{r}=r \pi} f^{i, r, r .}(\lambda, T, u, v) \cos \theta_{r} d \Omega_{r}}{\int_{\Pi_{r}=r \pi} \cos \theta_{r} d \Omega_{r}}
$$

Finally, the emissivity is expressed in Eq. (7) as a function of the bidirectional reflectivity and the diffusion factor as follows.

$$
\varepsilon^{i}(\lambda, T, u, v)=1-\pi \eta_{d}^{i, r_{0}}(\lambda, T, u, v) \rho^{i, r} \cdot(\lambda, T, u, v)
$$

The Eq. (7), assumes that the direction of emission of the light is the same than the direction of observation. This condition is impossible to obtain experimentally because it would involve the light source to be inside the lens of the camera. This difficulty can be bypassed thanks to Helmholtz theorem. In the case of our technique, it has an implication on the diffusion factor as : $\eta_{d}^{i, r 0}(\lambda, T, u, v)=\eta_{d}{ }^{r 0, i}(\lambda, T, u, v)$.

This condition allows us to put the light source in another direction than the direction of observation. The measurement of the radiance temperature can be done in a first direction, and the reflectivity source can emit from another direction. The important constraint is that both measures have to be done in the same direction.

From equation(7), the relation (2) becomes (assuming that radiance temperature measurement is done in the observation direction $\left.r_{0}\right)$ :

$$
L^{0}\left(\lambda, T_{L}^{r}, u, v\right)=\left(1-\pi \eta_{d}^{i, r} \cdot(\lambda, T, u, v) \rho^{i, r \cdot}(\lambda, T, u, v)\right) L^{0}(\lambda, T, u, v)
$$

Even if the system is written for two wavelengths, the number of unknowns is always too much brought up. Our solution to this difficulty is to assume that the diffusion factor doesn't vary for two near wavelengths.

$$
\eta_{d}^{i, r_{0}}(\lambda, T, u, v) \rightarrow \eta_{d}^{i, r} \cdot(T, u, v)
$$

For opaque materials, this assumption is valid and has been verified several times [10] establishing the BDRF study of the material. The reflexion behaviour of a ray of light will depend a lot on its wavelength; but, for two near wavelengths, the global behaviour (analog spatial shape) will be the same. The shape of the reflexion on a diffuse surface depends most of all on the texture of the material. In this technique and for the two working wavelengths, the diffusion factor is considered like only geometric. The spectral variations are absorbed by the bidirectional reflectivity, which is measured for a very small solid angle. No hypothesis are made about the emissivity, it can vary with the wavelength, contrarily to the classical bicolor thermography. The simultaneous measurements of the radiance temperature and the bidirectional reflectivity is explained below.

\subsection{Measurement of the radiance temperature}

For a radiometric point of view, the camera converts the radiance intensity to gray level values. For a black body source, the radiance intensity is related to the radiance temperature by using the Planck's law (see Eq. (1)). In the NIR spectral band (from $0.75 \mu \mathrm{m}$ to $1.7 \mu \mathrm{m}$ ), the Planck's law can be replaced by the Wien's law. The radiometric model describes the relation between the radiance temperature and the gray level value delivered by the camera. In the NIR spectral band, a specific radiometric model, based on the introduction an effective wavelength which depends on temperature, is required [11] and is given by equations (10) and (11).

$$
I_{D}^{r_{0}}(\lambda, T, u, v)=A(u, v) \exp \frac{-C_{2}}{\lambda_{x} T_{L}} \quad \text { (10) with } \quad \frac{1}{\lambda_{x}}=a_{0}+\frac{a_{1}}{T_{L}}+\frac{a_{2}}{T_{L}^{r}}
$$

The model depends on four parameters that are determined during a calibration process which requires known reference temperatures provided by a black body cavity. 
It should be noted that for a low temperature range $\left(\# 100^{\circ} \mathrm{C}\right)$ and for a narrow spectral range (\#100nm), the use of a first order expression of the effective wavelength is sufficient. A second order expression is required for large temperature and spectral range.

The radiometric model allows a radiance temperature map to be computed by reversing equation (10).

\section{4.}

\section{Measurement of the bidirectional reflectivity}

According to reflectivity considerations, the camera converts the radiance intensity reflected by the object illuminated, to gray level values. The bidirectional reflectivity model is a linear model versus the reflected intensity. During a calibration process which requires reflectance reference, the proportionality parameter between the gray levels and bidirectional reflectivities is obtained (see Eq. (12) and (13)). The material used as a reference in this article is the Spectralon ${ }^{\circledR}$ whose bidirectional reflectivity is known and equal to 0.394 for our wavelengths.

$$
\rho^{i, r_{0}}(\lambda, T, u, v)=K_{r e f}(\lambda, T, u, v) I_{D}^{i, r_{0}}(\lambda, T, u, v)(12) \quad \text { with } \quad K_{r e f}(\lambda, T, u, v)=\frac{\rho_{r e f}^{i, r}(\lambda, T, u, v)}{I_{D r e f}^{i, r_{0}}(\lambda, T, u, v)}
$$

It should be noted that the parameter $\mathrm{K}_{\text {ref }}$ is specific for a given configuration, hence the experimental set-up must be the same for the step of calibration and measurement.

\subsection{Conclusion}

Finally, the main equation (8) can be written back for two wavelengths. Under the assumption (9) and knowing the radiance temperature and the bidirectional reflectivity, a system with two wavelengths can be solved by computing the diffusion factor and the true temperature as follows:

$$
\begin{aligned}
& L^{0}\left(\lambda_{1}, T_{L 1}^{r_{0}}, u, v\right)=\left(1-\pi \eta_{d}^{i, r_{0}}(T, u, v) \rho^{i, r_{0}}\left(\lambda_{1}, T, u, v\right)\right) L^{0}\left(\lambda_{1}, T, u, v\right) \\
& L^{0}\left(\lambda_{r}, T_{L 2}^{r .}, u, v\right)=\left(1-\pi \eta_{d}^{i, r_{0}}(T, u, v) \rho^{i, r .}\left(\lambda_{2}, T, u, v\right)\right) L^{0}\left(\lambda_{2}, T, u, v\right)
\end{aligned}
$$

For $\lambda_{1}<\lambda_{2}$, two cases of resolution can be approached: (a) if $\rho 1=\rho 2$, the colour temperature, used in bicolour measurements, is found:

$$
T_{C}=T_{L \square} T_{L 2} \frac{\left(\lambda_{1}-\lambda_{2}\right)}{\lambda_{1} T_{L 1}-\lambda_{2} T_{L 2}}
$$

The colour temperature is then equal to the true temperature. (b) if $\rho 1$ is not equal to $\rho 2$, the theory suggests that there are cases where no solution exists. In our experiment, that possibility has never been encountered.

\section{Experimental set-up and results}

3.1.

\section{Presentation of the apparatus}

As mentioned in the introduction, the NIR spectral band provides the best trade-off between the temperature range and the emissivity dependence. The choice of both working wavelengths is on one hand motivated for the optimization of the received flux by the camera to improve the camera detectability, and on the other hand in respect to the hypothesis on the independence of the diffusion factor with regard to the wavelength. The choice of the specific working wavelengths of 1,3 and $1.55 \mu \mathrm{m}$ satisfies this double requirement which so express: the wavelengths must be the highest and the closest possible. Hence, the experimental setup used in order to validate the proposed method is illustrated in Fig. (1). The setup is composed of three elements:

(a) A NIR camera to perform the radiance temperature and reflectivity measurements: The camera exhibits a broad spectral response from $0,4 \mu \mathrm{m}$ to $1,7 \mu \mathrm{m}$. The detector is a modified InGaAs FPGA chip which is composed by $320^{\star} 256$ pixels what allows a good spatial resolution. To control the emitted and the reflected flux, the exposure time is controlled and it varies from $1 \mu \mathrm{s}$ up to $1 \mathrm{~s}$. The camera is equipped by filters with a centered wavelength of 1,3 and $1,55 \mu \mathrm{m}$ and broad spectral width of $100 \mathrm{~nm}$. The bandwidth is the best trade off between the level of the radiance intensity and the respect of the assumption that the Eq. (9) is right in monochromatic conditions. 
(b) Two laser sources operating at 1,3 and 1,55 $\mu \mathrm{m}$, to provide light required for the reflection measurements. The choice of the power is realized so that the flux reflected is in the same order than the thermal flux. The power is then $20 \mathrm{~mW}$ maximum, emitted continuously. The laser radiation passes through optics fibers before being collimated and emitted at about 1 meter from the object. The two lasers have to impact exactly the same point on the object. A mechanical micro metric precision apparatus have been mounted on the optical bench to fulfil this condition.

(c) A hot body with different emissivities (the object under study). This object is a planar copper sheet sizing about $100 \mathrm{~mm} \times 100 \mathrm{~mm}$, with four different emissivity areas. The object can be heated at several temperature levels ranging from 200 to $600^{\circ} \mathrm{C}$. A thermocouple is placed in the thickness of the plate to provide a reference temperature measurement.
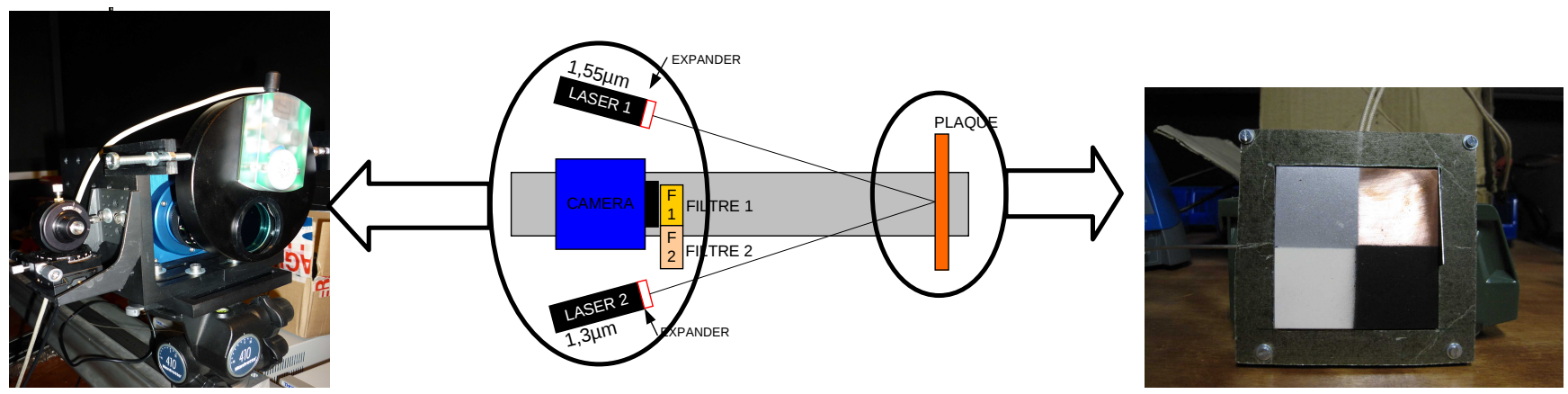

Fig. 1. Thermoreflectometry apparatus and the representative target

3.2.

Results

A first experiment of true temperature measurement, called thermoreflectometry, has been performed with the plate heated at a temperature of $240^{\circ} \mathrm{C}$ (set point imposed to the controlled heating device). The radiance temperature is obtained thanks to the radiometric model (see section 1.3). The bidirectional reflectivity measurement is performed using the reflectometry technique applied to each area of the plate and for a unique point. Then the true temperature and the diffusion factor are computed from the fusion of these two parameters (see Eq. (14)).

\subsection{1.}

\section{Radiance temperature measurements}

Fig(2) shows the radiance temperature of the plate heated at a temperature of $240{ }^{\circ} \mathrm{C}$, at the wavelength of $1,3 \mu \mathrm{m}$ and thanks to model (10) and (11) at first order (The same figure could be plotted for wavelength $1,55 \mu \mathrm{m}$ ). This image shows that the four areas of the plate do not exhibit the same radiance temperature due to different emissivity values. The values for one pixel are computed in table 1.

The radiance temperature of the area painted in black is the highest because it presents the strongest emissivity. On the contrary, the one painted in silver with low emissivity presents a lower radiance temperature. As illustrated in Fig. (3), the colour temperature, computed with the radiance temperatures (Eq. (15)), is not the same for all the areas of the plate. The silver and the copper areas seem to show a kind of texture, whereas black and white areas are a lot more homogenous. It might seem, therefore, that the assumption of the independence of the emissivity with the wavelength is not verified. We shall confirm this report in the following paragraph with various points of reflectivity measurement.

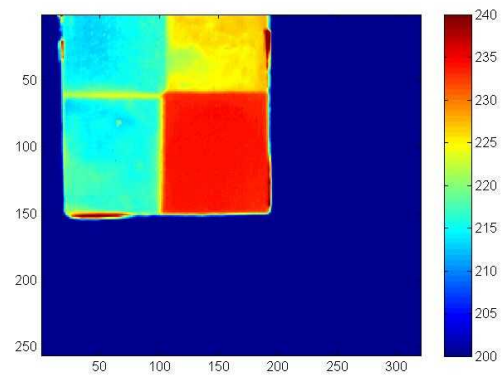

Fig. 2. Image of the radiance temperature at wavelength of $1,3 \mu \mathrm{m}$

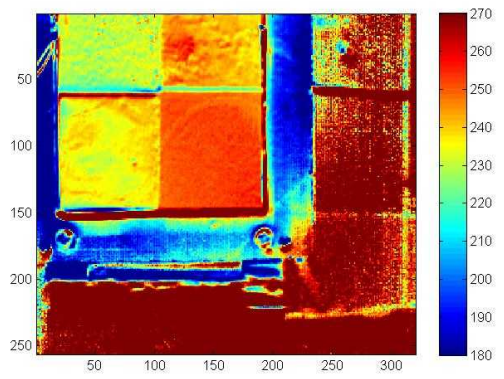

Fig. 3. Image of the colour temperature of the plate

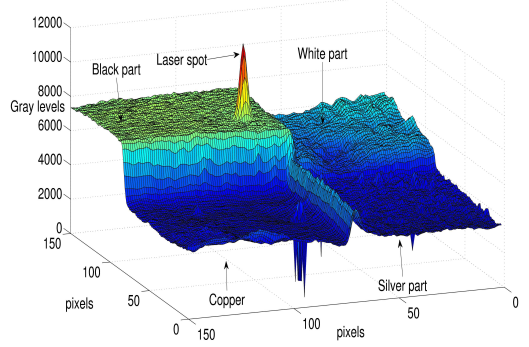

Fig. 4. Image of the laser impacting the heated plate (numerical levels) 


\subsection{2.}

\section{Bidirectional reflectivity measurements}

Fig.(4) shows a 3D view of the impact of the laser on the plate. To retrieve only the contribution of the laser, (and to suppress thermal contribution), subtraction of images with laser and without lasers have been done. From this subtracted image, bidirectional reflectivity is obtained thanks to (Eq. (12)) and calibration coefficient (Eq. (13)). The bidirectional reflectivity is measured on a unique pixel, where the spot's intensity is maximum. In table 1 , the values of the bidirectional reflectivity are tabulated for each area of the plate and for the two wavelengths. The value of the bidirectional reflectivity can be interpreted as a indication for the reflexion behaviour of the surface. The silver and copper, have the strongest bidirectional reflectivity for both wavelengths. As they are metallic compounds, they have a specular behaviour. White and black paint are more diffuse. Moreover, the value is different for each wavelength. This proves that this quantity is highly spectral dependant and can not be considered and constant (grey body behaviour).

\subsubsection{Determination of true temperature and diffusivity factor}

From the radiance temperatures and the bidirectional reflectivities for each wavelength and each area, the true temperature can be computed by solving the system of Eq. (14). Fig. (5) illustrates the determination of true temperature, for a specific laser position on the plate, which is the convergence point of the radiance and colour temperatures versus the diffusion factor.
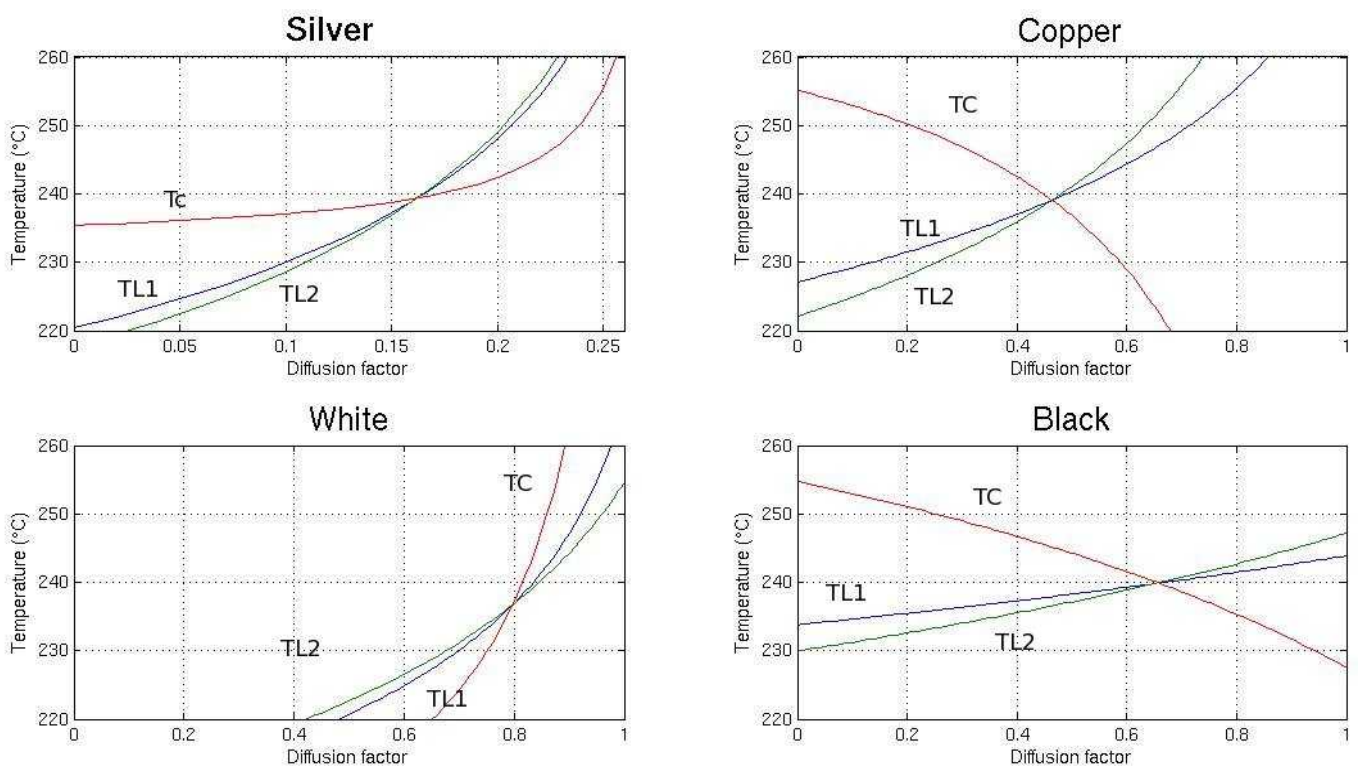

Fig. 5. Convergence temperatures for the four areas

The convergence temperature and the diffusion factor values are shown in table 1 for each area of the plate. The true temperature of the plate is $240^{\circ} \mathrm{C}$, given by the thermocouple mounted on it. The results shown are computed for a unique pixel and for each area of the plate.

\begin{tabular}{|l|l|l|l|l|}
\hline & \multicolumn{1}{|c|}{ White polish } & \multicolumn{1}{c|}{ Silver paint } & \multicolumn{1}{c|}{ Copper } \\
\hline$T_{\mathrm{L} 1}\left({ }^{\circ} \mathrm{C}\right)$ & 207.53 & 220.41 & 233.80 & 227.14 \\
\hline$T_{\mathrm{L} 2}\left({ }^{\circ} \mathrm{C}\right)$ & 209.28 & 215.83 & 229.96 & 222.10 \\
\hline$T_{\mathrm{C}}\left({ }^{\circ} \mathrm{C}\right)$ & 198.88 & 247.75 & 256.03 & 188.58 \\
\hline$\rho^{\mathrm{r} 0, \mathrm{i}}\left(\lambda_{1}, \mathrm{~T}\right)\left(\mathrm{sr}^{-1}\right)$ & 0.292 & 1.106 & 0.110 & 0.276 \\
\hline$\rho^{\mathrm{r} 0, \mathrm{i}}\left(\lambda_{2}, \mathrm{~T}\right)\left(\mathrm{sr}^{-1}\right)$ & 0.257 & 1.083 & 0.145 & 0.317 \\
\hline$T^{*}\left({ }^{\circ} \mathrm{C}\right)$ & 248.43 & 234.80 & 241.48 & 239.07 \\
\hline$\eta_{\mathrm{d}}$ & 0.8914 & 0.1383 & 0.7476 & 0.4641 \\
\hline
\end{tabular}




\begin{tabular}{|l|l|l|l|l|}
\hline$\varepsilon_{1}$ & 0.180 & 0.523 & 0.736 & 0.437 \\
\hline$\varepsilon_{2}$ & 0.245 & 0.501 & 0.663 & 0.486 \\
\hline
\end{tabular}

Table 1. True temperatures, diffusion factors and spectral emissivities for the four areas

When compared to the thermocouple temperature $\left(240^{\circ} \mathrm{C}\right)$, the convergence temperature is getting very near the true temperature for each area. The maximum absolute error is obtained for the white area, and is equal to $8.43^{\circ} \mathrm{C}$. In comparison of the colour temperature, the error is very low. The maximum error of the colour temperature is equal to $51.42{ }^{\circ} \mathrm{C}$ for the copper. The technique of thermoreflectometry is then more robust that 2-colour thermography and is also able to measure temperature for materials with low emissivity.

The values of the diffusion factor are representative of the reflexion behaviour of the material. Following definition (6), the diffusion factor must be equal to 1 for a perfect diffusor, and equal to 0 for a perfect specular surface. In our case, the silver area exhibits the lowest diffusion factor, due to its specular behaviour. The white polish shows the highest diffusion factor, so it has the most diffuse behaviour.

About the emissivity values, the black area emits the most for each wavelength. The values can be correlated to the radiance temperatures which are the highest also for the black paint. The white polish emits the less and exhibits the smallest radiance temperature. Furthermore, we can note that the emissivities are always different for the two wavelengths. The lowest relative difference between the two values is obtained for the silver and it is equal to $4.2 \%$. This area seems the most like a grey body. As a consequence, its colour temperature is the nearest from the true one.

Thermoreflectometry technique is able to measure emissivities of materials and could help to build databases. It also gives the true temperature of a hot body without contact and is more robust to spectral variations than the classical 2-colour thermography.

\subsubsection{Non-homogeneity of test areas}

A second experiment has been carried out in order to demonstrate the advantage of a field measurement of bidirectional reflectivities. In this objective, the lasers have impacted 16 times each part of the plate. In table 2, the relative standard deviations around a mean value of all the pixels belonging to an area are tabulated for the radiance temperature and the bidirectional reflectivities.

\begin{tabular}{|c|c|c|c|c|}
\hline & $\begin{array}{l}\text { Radiance temperature } \\
\text { variation at } \lambda_{1}\end{array}$ & $\begin{array}{l}\text { Radiance temperature } \\
\text { variation at } \lambda_{2}\end{array}$ & $\begin{array}{l}\text { Bidirectional reflectivity } \\
\text { variation at } \lambda_{1}\end{array}$ & $\begin{array}{l}\text { Bidirectional } \\
\text { variation at } \lambda_{1}\end{array}$ \\
\hline White polish & $0.89 \%$ & $2.81 \%$ & $1.60 \%$ & $2.11 \%$ \\
\hline Silver paint & $0.59 \%$ & $2.62 \%$ & $9.29 \%$ & $28.55 \%$ \\
\hline Black paint & $0.54 \%$ & $2.37 \%$ & $3.02 \%$ & $2.57 \%$ \\
\hline Copper & $0.88 \%$ & $3.18 \%$ & $15.93 \%$ & $30.56 \%$ \\
\hline
\end{tabular}

Table 2. Variations of radiance temperature and bidirectional reflectivity for the four parts

In the case of the black and the white parts, a small variation of the radiance temperature and the bidirectional reflectivities is observed. Indeed, the state of surface is homogeneous and the behaviour remains mainly diffuse which make the measurement of the bidirectional reflectivity easier. In that case, the computation of mean value of the bidirectional reflectivity gives a correct true temperature. On the other hand, in the case of the copper part, the dispersion around the mean value of the radiance temperature and the bidirectional reflectivity is very strong. An oxide layer has occurred and its apparition is not homogeneous. In that case, a field measurement of the bidirectional reflectivity can only give a correct true temperature for every pixel of the image. At the end, the case of the silver, is more complicated because its specular behaviour introduces a strong variation of the reflectivity. When the laser spot changes its position on the silver part, the specular direction also changes. Like the camera doesn't move, it detects a change in the bidirectional reflectivity. This can explain the variation of bidirectional reflectivity. Both the radiance temperature and reflectivity measurements become highly geometrically dependant. A measurement for a specular material represents a borderline case for our study.

\section{Conclusion}


The thermoreflectometry technique intends to measure the true temperature field of an object by fusing simultaneously, on line, radiance temperature and bidirectional recflectivities measurements for two wavelengths. These measurements are achieved with a NIR infrared camera coupled with two laser sources. The radiance temperature measurement, done for two wavelengths, is accurate to within $0.5 \%$ thanks to a specific radiometric model. The bidirectional reflectivity measurement, done for two wavelengths, is validated on a reduced surface represented by the impact of the spot laser. The results testify to the potentiality and the accuracy of the technique and suggest that the measurement on a point is a borderline study case. To put it more precisely, for homogeneous surfaces in thermal equilibrium conditions, the bidirectional reflectivity measurement is performed in different points with low spatial variations. In this case, a punctual measurement is sufficient and its mean value provides a realistic true temperature measurement. Nevertheless, for material with rough surfaces, a field measurement is an optimal solution. Our first results show the sensibility of the technique to the texture of the material and quite the potential for fields measurement of the dense true temperature. In contrast, for specular surfaces, the accuracy of the bidirectional reflectivity measurement is difficult.

For an opaque material with a diffuse surface, the technique allows the bidirectional reflectivity to be tracked on line and the true temperature to be determined without the emissivity knowledge. The results provided on a plate with different emissivities have argued that true temperature of materials can be retrieved with a high accuracy and sensitivity to the variation of the emissivity. The assumption of a reflection indicator independent from the wavelength seems to be more realistic than an emissivity independent from the wavelength in the case of bicolour thermography. The accuracy of colour temperature results does illustrate that the hypothesis of the grey body behaviour is not sufficient.

The further investigations will help enhance the efficiency of the punctual reflectivity measurement by using a pattern projected on the object. Thanks to new Leds sources, powerful enough to be detectable among the thermal flow and wide enough to cover a relatively wide surface, a field measurement of the reflectivity may eventually be possible.

\section{REFERENCES}

[1], Herve P.; Viellard L., Morel A., "Radiométrie : L'ultraviolet (Radiometry : the ultraviolet)", Revue pratique de contrôle industrie, 1997.

[2] F. Meriaudeau, "Real time multispectral high temperature measurement: Application to control in the industry", Image and Vision Computing, volume 25, Issue 7, July 2007, Pages 1124-1133.

[3] Traverse J.P., Badie J.M., "Mesure des Températures par pyrométrie optique à l'aide d'un rayonnement laser". I Ed. Ampère, Paris/, 1976, pp. 9-14.

[4] Kunz H., "On the state of ratio pyrometry with laser absorption measurements", /Temperature Measurement: Inst.Phys. Conf.Ser/ 26, ${ }^{*} * 1975$, pp.273-277.

[5], Murray T.P. "Polaradiometer-A new instrument for temperature measurements". / Rev. Sci. Instrum./ *38, *1967*, *pp. 791-798.

[6] M.D. Drury, K.D. Perry, T. Land 'Pyrometers for surface temperature measurements.' Journal of Iron and Steel Institute, pp. 245-250. 1951.

[7] D. Hernandez ' A concept to determine the true temperature of opaque materials using a tricolor pyroreflectometer' , Review of Scientific Instruments, Vol 76, 024904, pp1-7, 2005

[8] D. Hernandez, J.M Badie, F.Escourbiac, R. Reichle 'Development of two-colour pyroreflectometry technique for temperature monitoring of tungsten plasma facing components', Fusion Engineering and Design, Vol 83, pp 672-679, 2008.

[9] J. Behaghel, S. Berthier, and J. Lafait. Propriétés optiques des surfaces sélectives : définitions et méthodes de caractérisation.Physics/Physics archives, 1981.

[10]D. Hernandez, J.M. Badie, F. Escourbiac, and R. Reichle. 'Development of two-colour pyroreflectometry technique for temperature monitoring of tungsten plasma facing components'. Fusion Engineering and Design, 83 :672-679, May 2008.

[11]Y. Rotrou, T. Sentenac, Y. Le Maoult, P. Magnan \& J. Farré. Near infrared thermography with Silicon FPAComparison to MWIR and LWIR Thermography. QIRT Journal, vol.3, no. 1, pages 93-115, 2006. 